\title{
Cataland: Why the Fuss?
}

\author{
Christian Stump 收, Hugh Thomas啡, and Nathan Williams非 \\ ${ }^{1}$ Institut für Mathematik, Freie Universität Berlin, Germany \\ ${ }^{23}$ LaCIM, Université du Québec à Montréal, Montréal (Québec), Canada
}

\begin{abstract}
The main objects of noncrossing Catalan combinatorics associated to a finite Coxeter system are noncrossing partitions, sortable elements, and cluster complexes. The first and the third of these have known Fuss-Catalan generalizations. We provide new viewpoints for these, introduce a corresponding generalization of sortable elements as elements in the positive Artin monoid, and show how this perspective ties together all three generalizations.

Résumé. Les objets principaux de la combinatoire de Catalan non-croisée associés à un système de Coxeter fini sont les partitions non-croisées, les éléments triables, et les complexes de clusters. Le premier et le troisième d'entre eux ont des généralisations Fuss-Catlan connues. Nous fournissons de nouveaux points de vue pour ceux-ci, nous introdusons une généralisation des éléments triables comme éléments dans le monoïde positif d'Artin, et nous montrons comment cette perspective regroupe les trois généralisations.
\end{abstract}

Keywords. Coxeter-Catalan combinatorics, Fuß-Catalan numbers, noncrossing partitions, Coxeter-sortable elements, cluster complexes, associahedra, subword complexes

\section{Introduction}

Fix a finite Coxeter system $(W, \mathcal{S})$ and a (standard) Coxeter element $c \in W$-that is, a product of the simple generators $\mathcal{S}$ in any order. There are three noncrossing Catalan objects associated to this data [15, [16, 6, 13]:

- the $c$-noncrossing partitions $\mathrm{NC}(W, c)$;

- the $c$-sortable elements Sort $(W, c)$ (which generalize the 231-avoiding permutations); and

- the $c$-cluster complexes $\operatorname{Asso}(W, c)$ (which generalize the well-studied dual associahedron).

Although no uniform proof is currently known, these noncrossing families are counted by the Catalan number of type $W \operatorname{Cat}(W):=\prod_{i=1}^{n} \frac{h+d_{i}}{d_{i}}$, where $d_{1} \leq d_{2} \leq \ldots \leq d_{n}$ are the degrees and $h:=d_{n}=$ $\operatorname{ord}(c)$ is the Coxeter number of $W$. These noncrossing Catalan objects have two natural lattice structures:

- the $c$-noncrossing partition lattice $\mathrm{NCL}(W, c)$; and

- the $c$-Cambrian lattice $\operatorname{Camb}(W, c)$.

\footnotetext{
†Email: christian. stump@fu-berlin. de. Supported by the DFG grant STU 563/2 "Coxeter-Catalan combinatorics".

‡Email: hugh.ross.thomas@gmail.com. Partially supported by an NSERC Discovery Grant and the Canada Research Chairs program.

§Email: nathan.f.williams@gmail.com.
}

1365-8050 @ 2016 Discrete Mathematics and Theoretical Computer Science (DMTCS), Nancy, France 
The noncrossing partition lattices and the cluster complex have been generalized in the literature by introducing a nonnegative integral parameter $m$. Since many generalizations exist, we refer to this particular generalization as an m-eralization. The objects associated with these $m$-eralizations are counted by the Fuß-Catalan numbers of type $W$

$$
\mathrm{Cat}^{(m)}(W):=\prod_{i=1}^{n} \frac{m h+d_{i}}{d_{i}} .
$$

More precisely, D. Armstrong defined and studied the m-eralized c-noncrossing partitions $\mathrm{NC}^{(m)}(W, c)$ for any Coxeter element $c$ in [2], generalizing P. Edelman's construction from [9] to all finite Coxeter groups; and S. Fomin and N. Reading defined and studied the $m$-eralized $c$-cluster complex $\operatorname{Asso}^{(m)}(W, c)$ for a bipartite Coxeter element $c$ in [10].

There are several components missing in this story. The aim of this extended abstract is to complete the $m$-eralization of noncrossing Catalan objects using the spherical Artin group corresponding to the finite Coxeter system $(W, \mathcal{S})$. Due to space limitations, we will omit much of our discussion of noncrossing partitions, the cluster complexes, and connections to the representation theory of hereditary Artin algebras.

Coxeter-sortable elements. The most glaring omission is that no $m$-eralization of $\mathrm{N}$. Reading's $c$-sortable elements [16] has appeared in the literature. It is straightforward to extract a definition involving chains of sortable elements by combining D. Armstrong's definition of $m$-eralized $c$-noncrossing partitions and N. Reading's shard order (see Section 7p, but such a definition is unsatisfactory without $a$ corresponding $m$-eralization of the weak order. At the heart of this extended abstract is the observation that such an $m$ eralization is given by the interval $\left[e, w_{\circ}^{m}\right]$ in the weak order of the positive Artin monoid $B^{+}=B^{+}(W)$. These intervals have been previously studied by P. Dehornoy in an enumerative context [8].

Definition 1.1 $W^{(m)}:=\left\{w \in B^{+}(W): w \leq w_{\circ}^{m} \in B^{+}(W)\right\}$.

There is a natural bijection between the elements of $W$ and $W^{(1)} ; W^{(m)}$ is a rank-symmetric lattice under the weak order, recovering the usual weak order for $m=1$. In Section 4 , we provide the missing $m$-eralization of the $c$-sortable elements as a lift of $\mathrm{N}$. Reading's definition from $W$ to $W^{(m)}$.

Definition 1.2 Let $c \in W$ be a Coxeter element. An element $w \in W^{(m)}$ is $c$-sortable if the $c$-sorting word $\mathrm{w}(\mathrm{c})$ for $w$ yields a decreasing sequence of subsets of positions in $\mathrm{c}$. Denote the set of all such $m$-eralized $c$-sortable elements by Sort $^{(m)}(W, c)$.

We provide three characterizations of Sort ${ }^{(m)}(W, c)$ in Definition 3.4 Proposition 4.3, and in Definition 5.2, extending N. Reading's characterizations when $m=1$ [15, Sections 2 and 4].

Cluster complexes. The second missing component is that there is no simple combinatorial definition of the $m$-eralized $c$-cluster complex when the Coxeter element $c$ is not bipartite. In the full version of this work, we supply this definition with an $m$-eralized $c$-compatibility relation. We prove the existence and uniqueness of this relation using an $m$-eralization of the subword complex approach to $c$-cluster complexes given in [7, 13].

Definition 1.3 The simplicial complex $\operatorname{Asso}^{(m)}(W, c)$ is the set of subwords (as indices of positions) of the word $\mathrm{cw}_{\circ}^{m}(\mathrm{c})$ whose complements contain a word for $w_{\circ}^{m} \in W$ of length $m N$, where $N=\ell\left(w_{\circ}\right)$. 
We recover that $\mathrm{Asso}^{(m)}(W, c)$ is vertex-decomposable, and hence shellable. With the $m$-eralizations of noncrossing partitions, sortable elements, and subword complexes in hand, we prove the following theorem.

Theorem 1.4 There are explicit and uniform bijections between the three families

- the m-eralized c-noncrossing partitions $\mathrm{NC}^{(m)}(W, c)$;

- the m-eralized c-sortable elements Sort $^{(m)}(W, c)$; and

- the m-eralized c-cluster complexes $\operatorname{Asso}^{(m)}(W, c)$.

By "explicit", we mean that we provide a bijection, rather than just proving existence of a bijection by a counting argument. The term "uniform" specifies that the description of the bijection does not use the classification theorem of finite Coxeter systems.

In the following, we focus on the structure of $m$-eralized $c$-sortable elements $\operatorname{Sort}^{(m)}(W, c)$, for the full treatment and many more details, we refer to the full length article of this extended abstract.

Cambrian lattices. Although the exchange graph of S. Fomin and N. Reading's $m$-eralized $c$-cluster complex can be used to define a Cambrian graph for bipartite Coxeter elements, no corresponding poset has been considered in the literature for $m>1$. In particular, no orientation of the exchange graph was known to be a lattice. In Section 6, we close this third gap by defining the $m$-eralized $c$-Cambrian lattice on Sort ${ }^{(m)}(W, c)$. Our construction $m$-eralizes N. Reading's Cambrian lattices, which are themselves generalizations of the classical Tamari lattices.

Theorem 1.5 The restriction of the weak order to Sort ${ }^{(m)}(W, c)$ is a lattice.

We call this the $m$-eralized $c$-Cambrian lattice. One intuition comes from the isomorphism between shard order restricted to $c$-sortable elements and the noncrossing partition lattice: just as D. Armstrong considered component-wise absolute order on chains in $\operatorname{NCL}(W, c)$ [2], our $m$-eralized $c$-Cambrian lattices can be seen to be component-wise weak order on the corresponding chains in $\operatorname{Shard}(W)$.

In summary, we place the program of $m$-eralizing noncrossing Coxeter-Catalan combinatorics in the context of the corresponding positive Artin monoid.

\section{Background and the m-eralized weak order}

\subsection{Coxeter systems}

Let $(W, \mathcal{S})$ be a (finite) Coxeter system of rank $n=|\mathcal{S}|$. The elements in the set $\mathcal{S}$ are called simple generators or simple reflections. The set of reflections in $W$ is defined to be $\mathcal{R}:=\left\{s^{w}: s \in \mathcal{S}, w \in W\right\}$, where we write $u^{w}:=w u w^{-1}$ (i) We use the corresponding root system $\Phi=\Phi^{+} \sqcup \Phi^{-}$(so that $s \mapsto \alpha_{s} \in$ $\Phi^{+}$) without further comment. The spherical Artin system $(B(W), \mathcal{S})$ corresponding to the finite Coxeter system $(W, \mathcal{S})$ is the group $B(W)$ given by (a formal copy of) the generators $\mathcal{S}$ and the presentation

$$
B(W)=\left\langle\mathcal{S}:\left(s s^{\prime}\right)^{m\left(s, s^{\prime}\right) / 2}=\left(s^{\prime} s\right)^{m\left(s^{\prime}, s\right) / 2} \text { for } s, s^{\prime} \in \mathcal{S} \text { with } s \neq s^{\prime}\right\rangle .
$$

Rather than studying $B(W)$, we will restrict ourselves to the positive Artin monoid $B^{+}(W)$ (or simply $B^{+}$), which is the submonoid of $B(W)$ generated by $\mathcal{S}$.

(i) We choose this convention over the more natural choice $w^{-1} u w$ for reasons of brevity. 


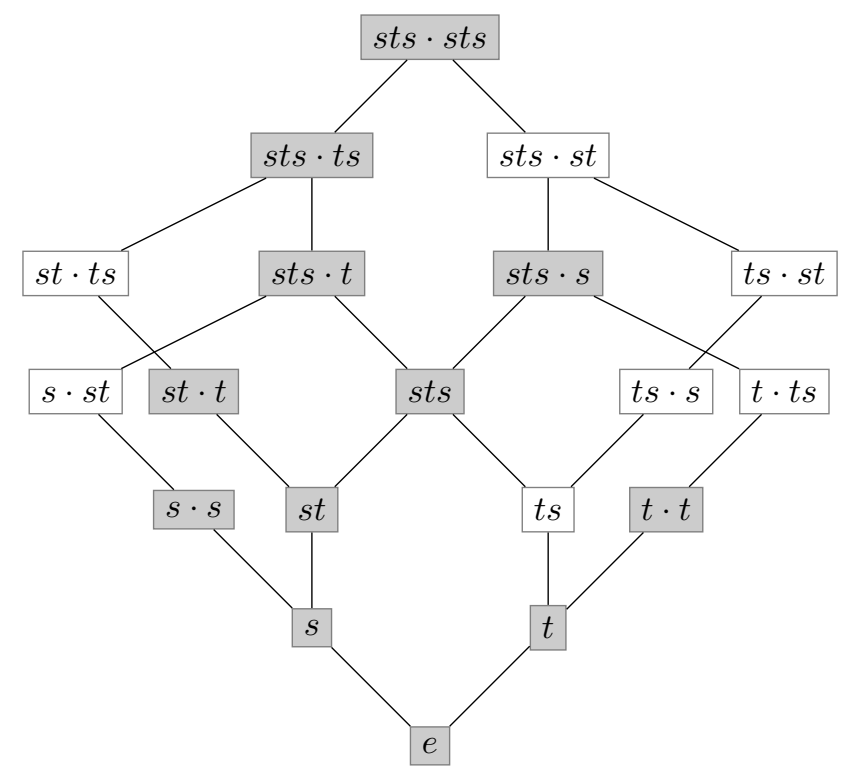

Fig. 1: Weak ${ }^{(2)}\left(A_{2}\right)$. The elements marked in gray are the $s t$-sortable elements.

\subsection{Weak order}

The (Coxeter) length of an element $w$ in the group $W$ or in the positive Artin monoid $B^{+}$is the length $\ell(w)$ of a shortest expression for $w$ as a product of the generators in $\mathcal{S}$. We define the support $\operatorname{supp}(w)$ for $w \in W$ or $w \in B^{+}(W)$ to be the set $\left\{s_{1}, \ldots, s_{p}\right\} \subseteq \mathcal{S}$ of simple reflections contained in any reduced word $\mathrm{s}_{1} \cdots \mathrm{s}_{p}$ for $w$. The (right) weak order on $W$ (resp. $B^{+}$) is the partial order Weak $(W)$ (resp. Weak $\left(B^{+}\right)$) defined by $u \leq w$ if there exists $v \in W$ (resp. $v \in B^{+}$) with $u v=w$ and $\ell(u)+\ell(v)=\ell(w)$. If $w$ has such a factorization $u v=w$, we say that $u$ left-divides $w$ and $v$ right-divides $w$. We may write $w v^{-1}$ for $u$ and $u^{-1} w$ for $v$, even in $B^{+}$. A letter $s \in \mathcal{S}$ is called initial (resp. final) in an element $w$ in the group $W$ or in the Artin monoid $B^{+}$, if $s$ left-divides (resp. right-divides) $w$. It is well-known that Weak $(W)$ and Weak $\left(B^{+}\right)$are both lattices.Observe that the Coxeter group $W$ injects into $B^{+}(W)$ as the interval $\left[e, w_{\circ}\right]_{\operatorname{Weak}\left(B^{+}\right)}$, where $w_{\circ}:=\operatorname{lcm}(\mathcal{S})$. This injection preserves the weak order, so that $\operatorname{Weak}(W)=\left[e, w_{\circ}\right]_{\operatorname{Weak}\left(B^{+}\right)}$. The (right) $m$-weak order is defined as the interval Weak ${ }^{(m)}(W):=$ $\left.\left[e, w_{\circ}^{m}\right]\right]_{\text {Weak }\left(B^{+}\right)}$. We denote the set of elements simply by $W^{(m)}:=\left\{w: w \in\right.$ Weak $\left.^{(m)}(W)\right\}$. Figure 1 illustrates the Hasse diagram of Weak ${ }^{(2)}\left(A_{2}\right)$.

\subsection{Descents, Garside factorizations, and inversions}

The left descent set, right descent set, left ascent set, and right ascent set of $w \in W$ or of $w \in B^{+}$ by $\operatorname{des}_{L}(w):=\{s \in \mathcal{S}: s$ left divides $w\}, \operatorname{des}_{R}(w):=\{s \in \mathcal{S}: s$ right divides $w\}, \operatorname{asc}_{L}(w):=$ $\mathcal{S} \backslash \operatorname{des}_{L}(w)$, and $\operatorname{asc}_{R}(w):=\mathcal{S} \backslash \operatorname{des}_{R}(w)$. For $w \in W$, the covered reflections and the covering reflections are given by $\operatorname{cov}_{\downarrow}(w):=\left\{s^{w}: s \in \operatorname{des}_{R}(w)\right\}$ and $\operatorname{cov}^{\uparrow}(w):=\left\{s^{w}: s \in \operatorname{asc}_{R}(w)\right\}$.

Each element $w \in B^{+}$has a normal form called Garside factorization garside $(w)$, which gives an 


\begin{tabular}{|c|c|c|c|c|c|c|c|}
\hline garside & $\mathrm{w}(\mathrm{st})$ & $\operatorname{des}_{L}$ & $\operatorname{des}_{R}$ & garside & $\mathrm{w}(\mathrm{st})$ & $\operatorname{des}_{L}$ & $\operatorname{des}_{R}$ \\
\hline$s \cdot s$ & $\mathbf{s t}|\mathbf{s t}| s t$ & $s$ & $s$ & $t s \cdot s t$ & st|st|st & $t$ & $t$ \\
\hline$s \cdot s t$ & st|st|st & $s$ & $t$ & $s t s \cdot s$ & st $|\mathbf{s t}| \mathbf{s t}$ & $s, t$ & $s$ \\
\hline$t \cdot t$ & st|st|st & $t$ & $t$ & $s t s \cdot t$ & st|st|st & $s, t$ & $s, t$ \\
\hline$t \cdot t s$ & st|st|st & $t$ & $s$ & $s t s \cdot s t$ & st|st|st & $s, t$ & $s, t$ \\
\hline$s t \cdot t$ & st|st|st & $s$ & $t$ & $s t s \cdot t s$ & st|st|st & $s, t$ & $s, t$ \\
\hline$s t \cdot t s$ & st|st|st & $s$ & $s$ & sts $\cdot s t s$ & st|st|st & $s, t$ & $s, t$ \\
\hline$t s \cdot s$ & st|st|st & $t$ & $s$ & & & & \\
\hline
\end{tabular}

Fig. 2: The 13 elements in $B^{+}\left(A_{2}\right)$ having exactly two Garside factors with their st-sorting word and their descent sets.

alternative description of the $m$-weak order. To this end, set $w_{1}=w$ and $v_{1}=\operatorname{gcd}\left(w_{1}, w_{\circ}\right)$. For $i=2,3, \ldots$, as long as $w_{i-1} \neq e$, let $w_{i}=v_{i-1}^{-1} w_{i-1}, \quad v_{i}=\operatorname{gcd}\left(w_{i}, w_{\circ}\right)$. Then $\operatorname{garside}(w)=$ $w^{(1)} \cdot w^{(2)} \cdots \cdot w^{(k)}$, where $w^{(i)}:=v_{i}$ and the degree $\operatorname{deg}(w)$ is defined to be $k$. By construction, every factor sits inside the interval $\left[e, w_{\circ}\right]$ and so can be treated as an element of $W$.

Proposition 2.1 A factorization $v_{1} \cdot v_{2} \cdots \cdot v_{k}$ with $v_{i} \in W$ is the Garside factorization of the element $w=v_{1} \cdots v_{k} \in B^{+}$if and only if $\operatorname{des}_{R}\left(v_{i-1}\right) \supseteq \operatorname{des}_{L}\left(v_{i}\right)$.

To emphasize different Garside factors, we may separate them by a centered dot. For $w \in W$, we write

$$
\operatorname{inv}(w):=\left\{\alpha_{s_{1}}, s_{1}\left(\alpha_{s_{2}}\right), \ldots, s_{1} \cdots s_{\ell-1}\left(\alpha_{s_{\ell}}\right)\right\} \subseteq \Phi^{+}
$$

A colored positive root is positive roots together with a color given by a nonnegative integer. A simple reflection $s \in \mathcal{S}$ acts on a colored positive root as $s\left(\beta^{(k)}\right)=[s(\beta)]^{(k)}$ if $\beta \neq \alpha_{s}$ and $s\left(\beta^{(k)}\right)=\beta^{(k+1)}$ if $\beta=\alpha_{s}$. Given any $\mathcal{S}$-word $\mathrm{Q}=\mathrm{s}_{1} \cdots \mathrm{s}_{p}$, The colored inversion sequence of $\mathrm{Q}$ is given by

$$
\operatorname{inv}(\mathrm{Q}):=\left(\beta_{1}^{\left(m_{1}\right)}, \ldots, \beta_{p}^{\left(m_{p}\right)}\right)
$$

where $\beta_{i}^{\left(m_{i}\right)}$ is given by $\mathrm{s}_{1} \cdots \mathrm{s}_{i-1}\left(\alpha_{s_{i}}^{(0)}\right)$.

\subsection{Coxeter elements}

A (standard) Coxeter element $c$ for $(W, \mathcal{S})$ and for $(B(W), \mathcal{S})$ is defined to be the product of the simple reflections $\mathcal{S}$ in any order. As the Dynkin diagram for a finite Coxeter group $W$ is a tree, we have that all Coxeter elements in $W$ are conjugate. We denote their common order by the Coxeter number $h$. The rank $n$ of $W$, the number $N=\ell\left(w_{\circ}\right)=\left|\Phi^{+}\right|$, and the Coxeter number are related by $N=n h / 2$.

Let $\mathrm{c}=\mathrm{s}_{1} \cdots \mathrm{s}_{n}$ be a particular reduced word for a Coxeter element $c$, and let $w$ be an element in $W$ (or in $B^{+}$). The c-sorting word $\mathrm{w}(\mathrm{c})$ for $w$ is then defined to be the lexicographically first subword of $\mathrm{c}^{\infty}=\left(\mathrm{s}_{1} \cdots \mathrm{s}_{n}\right)^{\infty}$ which is a reduced expression for $w$. To emphasize the different copies of $\mathrm{s}_{1} \cdots \mathrm{s}_{n}$, we may separate them by vertical bars. Finally, we write $\leq_{c}$ for the root order $\operatorname{inv}\left(\mathrm{w}_{\circ}(\mathrm{c})\right)$ on $\Phi^{+}$and analogously for the root order $\operatorname{inv}_{\mathcal{R}}\left(\mathrm{w}_{\circ}(\mathrm{c})\right)$ on $\mathcal{R}$. 


\section{Coxeter-sortable elements}

In this section, we review N. Reading's theory of $c$-sortable elements [14, 16, 15], recalling two characterizations of these elements. We then define an $m$-eralization of N. Reading's $c$-sortable elements as a certain subset Sort $^{(m)}(W, c)$ of $W^{(m)}$.

$\mathrm{N}$. Reading introduced and studied $c$-sortable elements in [14, 15]. The $c$-sortable elements have at least three different characterizations, each of which is useful in different ways.

Definition 3.1 (N. Reading [14]) An element $w \in W$ is c-sortable if the c-sorting word $\mathrm{w}(\mathrm{c})$ for $w$ defines a decreasing sequence of subsets of positions in c. We denote the set of c-sortable elements by $\operatorname{Sort}(W, c)$. The $c$-Cambrian lattice $\mathrm{Camb}_{\text {Sort }}(W, c)$ is the restriction of $\operatorname{Weak}(W)$ to $\operatorname{Sort}(W, c)$.

Although the definition of being $c$-sortable depends on a particular choice of a reduced word c for the Coxeter element $c$, all $c$-sorting words $\mathrm{w}(\mathrm{c})$ are commutation equivalent and the property of being $c$-sortable does not depend on a particular chosen word.

The second characterization is the c-Cambrian recurrence, which is immediate from Definition 3.1

Proposition 3.2 (N. Reading [16, Lemma 2.1 and 2.2]) Let s be initial in $c$. Then

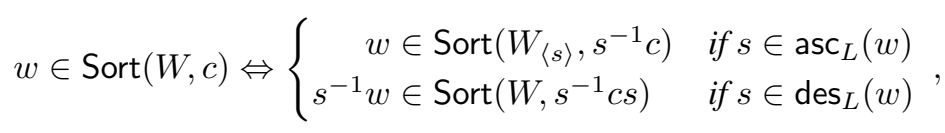

where $W_{\langle s\rangle}:=W_{S \backslash\{s\}}$.

N. Reading's third characterization describes $c$-sortable elements by their inversion sets as the $c$-aligned elements. We do not $m$-eralize this definition, and so omit further discussion.

\section{Lemma 3.3 (N. Reading [15, Corollary 4.5], [16, Theorem 1.2])}

1. For $u, v \in \operatorname{Sort}(W, c), \operatorname{inv}(u \wedge v)=\operatorname{inv}(u) \cap \operatorname{inv}(v)$. Furthermore, $u \wedge v \in \operatorname{Sort}(W, c)$.

2. Camb Sort $(W, c)$ is a sublattice of $\operatorname{Weak}(W)$.

We first define Sort ${ }^{(m)}(W, c)$ as elements in $W^{(m)}$ in analogy with Definition 3.1

Definition 3.4 An element $w \in W^{(m)}$ is c-sortable if the c-sorting word $\mathrm{w}(\mathrm{c})$ for $w$ defines a decreasing sequence of subsets of positions in c. We denote the set of c-sortable elements by $\operatorname{Sort}^{(m)}(W, c)$.

Example 3.5 In Weak ${ }^{(2)}\left(A_{3}\right)$ with $\mathrm{c}=s_{1} s_{2} s_{3}$, the element $w=s_{1} s_{2} s_{3} s_{1} s_{2} \cdot s_{3} s_{2} s_{1}$ has $c$-sorting word

$$
\mathrm{w}(\mathrm{c})=\left(\begin{array}{ccc|ccc|ccc|ccc}
1 & 2 & 3 & 1 & 2 & 3 & 1 & 2 & 3 & 1 & 2 & 3 \\
s_{1} & s_{2} & s_{3} & s_{1} & s_{2} & s_{3} & - & s_{2} & - & s_{1} & - & -
\end{array}\right) .
$$

It is not $c$-sortable since $s_{1}$ occurs in the fourth but not the third copy of c.

\section{Cambrian rotation and the Cambrian recurrence}

In this section, we generalize Proposition 4.3 to provide an inductive characterization of the $m$-eralized sortable elements. 
The Cambrian rotation and the Cambrian recurrence both depend on an operation Shift ${ }_{s}$ for an initial simple reflection $s$ in a Coxeter element $c$. The map Shift $s: \operatorname{Sort}^{(m)}(W, c) \rightarrow \operatorname{Sort}^{(m)}\left(W, s^{-1} c s\right)$ is defined for $w \in \operatorname{Sort}^{(m)}(W, c)$ by

$$
\operatorname{Shift}_{s}(w)=\left\{\begin{array}{ll}
w \vee s^{m} & \text { if } s \in \operatorname{asc}_{L}(w) \\
s^{-1} w & \text { if } s \in \operatorname{des}_{L}(w)
\end{array} .\right.
$$

It is nontrivial to show that this operation yields an element in $\operatorname{Sort}^{(m)}\left(W, s^{-1} c s\right)$.

We may now compose the shift operations in the order specified by any reduced $\mathcal{S}$-word for the Coxeter element $c$. This composition does not depend on the chosen reduced word, since two shifts Shift ${ }_{s}$ and Shift $_{t}$ commute for commuting $s, t \in \mathcal{S}$.

Definition 4.1 Let c be a Coxeter element. The $m$-eralized $c$-Cambrian rotation Camb $_{c}: \operatorname{Sort}^{(m)}(W, c) \rightarrow$ Sort $^{(m)}(W, c)$ is given by $\mathrm{Camb}_{c}=$ Shift $_{s_{n}} \circ \cdots \circ \mathrm{Shift}_{s_{1}}$ for any reduced $\mathcal{S}$-word $\mathrm{s}_{1} \mathrm{~s}_{2} \cdots \mathrm{s}_{n}$ for $c$.

Example 4.2 In Sort ${ }^{(2)}\left(A_{2}, s t\right)$, one orbit of $m$-eralized Cambrian rotation is given by

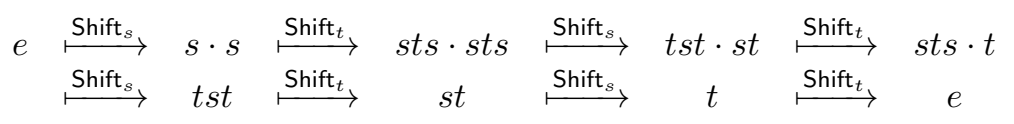

After removing the initial $e$, which is thought of as an element of Sort ${ }^{(2)}\left(A_{2}, s t\right)$, the right column consists of elements of Sort ${ }^{(2)}\left(A_{2}, s t\right)$, while the left column contains elements of Sort ${ }^{(2)}\left(A_{2}, t s\right)$.

Rather than take the join with $s^{m}$ in the first case of the definition of the shift operator, we could have sent $w$ to itself, viewed as an element of a parabolic subgroup. As an $m$-eralization of Proposition 3.2. we call this process the $m$-eralized $c$-Cambrian recurrence.

Proposition 4.3 Let s be initial in a Coxeter element $c$. Then

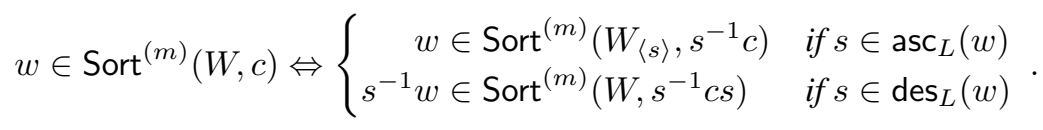

Example 4.4 Consider $s t s \cdot s \in \operatorname{Sort}^{(2)}\left(A_{2}, s t\right)$. Then the $m$-eralized $c$-Cambrian recurrence is computed as

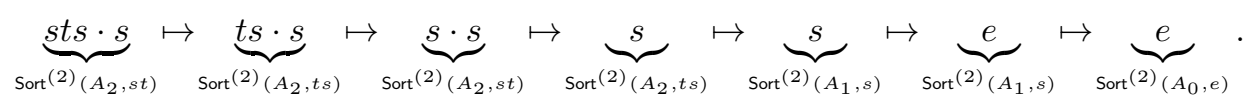

Figure 3 shows all 12 elements of Sort ${ }^{(2)}\left(A_{2}, s t\right) \cong \operatorname{Sort}_{\text {shard }}^{(2)}\left(A_{2}, s t\right)$, with their support. The notion Sort ${ }_{\text {shard }}^{(2)}\left(A_{2}, s t\right)$ will be defined and studied in Section 7.

\section{Factorwise Coxeter-sortable elements}

We now give an alternative description of $m$-eralized Coxeter-sortable elements using Garside factors. Let $w \in W$, let $c=s_{1} s_{2} \cdots s_{n}$ be a Coxeter element, and let $\operatorname{des}_{R}(w)=\left\{s_{i_{1}}, s_{i_{2}}, \ldots, s_{i_{k}}\right\}$, where $s_{i_{1}}^{w} \leq_{c} s_{i_{2}}^{w} \leq_{c} \cdots \leq_{c} s_{i_{k}}^{w}$ in the root order associated to c. Define the restriction of $c$ with respect to the 


\begin{tabular}{c|c|c|c|c} 
garside $(w)$ & Sort $^{(2)}\left(A_{2}, s t\right)$ & Sort $_{\text {shard }}^{(2)}\left(A_{2}, s t\right)$ & $\mathcal{C}_{c}(w)$ & $\operatorname{supp}(w)$ \\
\hline$e$ & $\mathrm{st}|\mathrm{st}| \mathrm{st}$ & $e \succeq e$ & $\alpha^{(0)}, \beta^{(0)}$ & - \\
$s t s \cdot t s t$ & $\mathrm{st}|\mathrm{st}| \mathrm{st}$ & $s t s \succeq s t s$ & $\alpha^{(2)}, \beta^{(2)}$ & $s, t$ \\
$s t s \cdot t$ & $\mathrm{st}|\mathrm{st}| \mathrm{st}$ & $s t s \succeq s$ & $\gamma^{(1)}, \alpha^{(2)}$ & $s, t$ \\
$s t$ & $\mathrm{st}|\mathrm{st}| \mathrm{st}$ & $s t \succeq e$ & $\beta^{(0)}, \gamma^{(1)}$ & $s, t$ \\
\hline$s$ & $\mathrm{st}|\mathrm{st}| \mathrm{st}$ & $s \succeq e$ & $\gamma^{(0)}, \alpha^{(1)}$ & $s$ \\
$t \cdot t$ & $\mathrm{st}|\mathrm{st}| \mathrm{st}$ & $t \succeq t$ & $\alpha^{(0)}, \beta^{(2)}$ & $t$ \\
$s t s \cdot t s$ & $\mathrm{st}|\mathrm{st}| \mathrm{st}$ & $s t s \succeq s t$ & $\beta^{(1)}, \gamma^{(2)}$ & $s, t$ \\
$s t s$ & $\mathrm{st}|\mathrm{st}| \mathrm{st}$ & $s t s \succeq e$ & $\alpha^{(1)}, \beta^{(1)}$ & $s, t$ \\
\hline$t$ & $\mathrm{st}|\mathrm{st}| \mathrm{st}$ & $t \succeq e$ & $\alpha^{(0)}, \beta^{(1)}$ & $t$ \\
$s t \cdot t$ & $\mathrm{st}|\mathrm{st}| \mathrm{st}$ & $s t \succeq s t$ & $\beta^{(0)}, \gamma^{(2)}$ & $s, t$ \\
\hline$s \cdot s$ & $\mathrm{st}|\mathrm{st}| \mathrm{st}$ & $s \succeq s$ & $\gamma^{(0)}, \alpha^{(2)}$ & $s$ \\
$s t s \cdot s$ & $\mathrm{st}|\mathrm{st}| \mathrm{st}$ & $s t s \succeq t$ & $\alpha^{(1)}, \beta^{(2)}$ & $s, t$
\end{tabular}

Fig. 3: All 12 elements of Sort ${ }^{(m)}\left(A_{2}, s t\right)$ and $\operatorname{Sort}_{\text {shard }}^{(m)}\left(A_{2}, s t\right)$, their skip sets and their supports, grouped into orbits under Cambrian rotation.

element $w$ to be the Coxeter element $\left.c\right|_{w}:=s_{i_{1}} s_{i_{2}} \cdots s_{i_{k}}$ of the Coxeter system given by the standard parabolic subgroup $W_{\operatorname{des}_{R}(w)}$ generated by the simple reflections in $\operatorname{des}_{R}(w)$. Observe that $\left.c\right|_{w}$ contains the same simple reflections as the restriction of $c$ to this standard parabolic subgroup, but that the order in which the simple reflections appear is not necessarily the order in which they appear in $c$-rather, it is the order coming from the root order associated to $c$.

Example 5.1 Consider type $A_{3}$ with $c=s_{1} s_{2} s_{3}$ and let $w=s_{1} s_{2} s_{3} s_{2}=s_{1} s_{3} s_{2} s_{3}$, so that $\operatorname{des}(w)=$ $\left\{s_{2}, s_{3}\right\}$. Since $s_{3}^{w^{(1)}}=(13) \leq_{c}(34)=s_{2}^{w^{(1)}}$, we then have $\left.c\right|_{w}=s_{3} s_{2}$.

Definition 5.2 Let $w$ be an element in $W^{(m)}$ with garside $(w)=w^{(1)} \cdot \cdots \cdot w^{(m)}$. We say that $w$ is factorwise $c$-sortable if $w^{(i)} \in W_{\operatorname{des}_{R}\left(w^{(i-1)}\right)}$ is $c^{(i)}$-sortable for all $1 \leq i \leq m$, where $w^{(0)}:=w_{\circ}$, $c^{(0)}:=c, c^{(i)}:=\left.c^{(i-1)}\right|_{w^{(i-1)}}$. We denote the set of factorwise c-sortable elements by Sort ${ }_{\text {fact }}^{(m)}(W, c)$.

Corollary 5.3 An element $w \in W^{(m)}$ is c-sortable if and only if it is factorwise c-sortable.

The next theorem transports the characterization of weak order on inversion sets to sortable elements, and generalizes Lemma 3.3.1].

Theorem 5.4 For $w, u \in \operatorname{Sort}^{(m)}(W, c), w \leq u$ if and only if $\operatorname{inv}(w) \subseteq \operatorname{inv}(u)$, and $\operatorname{inv}(w \wedge u)=$ $\operatorname{inv}(w) \cap \operatorname{inv}(u)$.

Remark 5.5 The first part of Theorem 5.4 does not hold in general for non-sortable elements, although it is true when $m=1$. The second part doesn't even hold for non-sortable elements when $m=1$.

\section{$6 m$-eralized Cambrian lattices}

Generalizing the case for $m=1$, we prove that $\operatorname{Sort}^{(m)}(W, c)$ is a sublattice of Weak ${ }^{(m)}(W)$. N. Reading's $c$-sortable elements are the key to understand certain order congruences on the weak order that respect the lattice structure of the weak order (and are therefore lattice congruences). We briefly summarize 


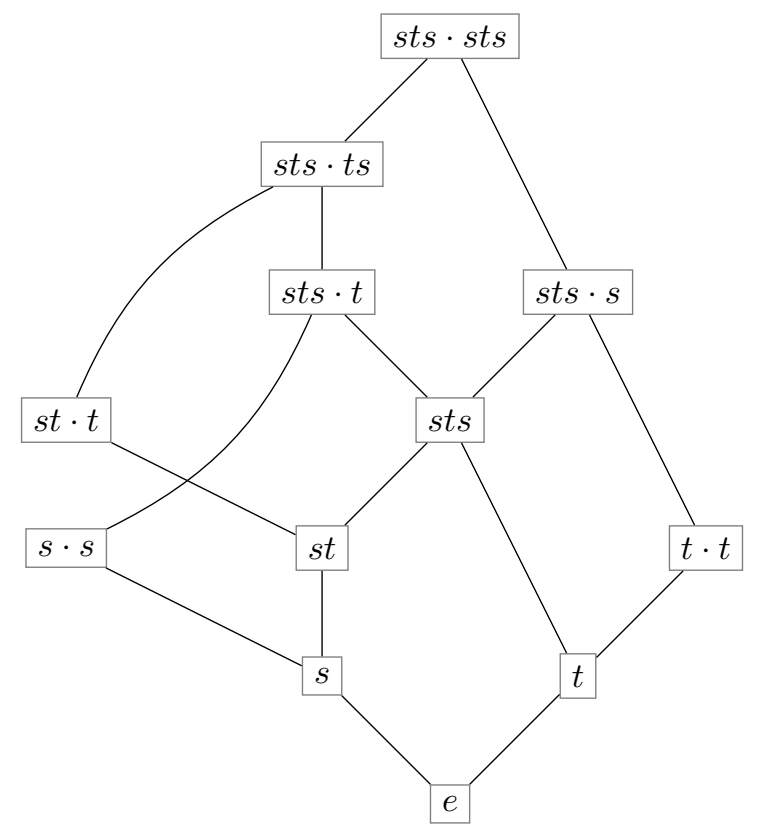

Fig. 4: $\mathrm{Camb}_{\text {Sort }}^{(2)}\left(A_{2}, s t\right)$.

some results of [16]. N. Reading defined an order-preserving projection $\pi_{\downarrow}^{c}:$ Weak $(W) \rightarrow \operatorname{Sort}(W, c)$ sending an element $w$ to the largest $c$-sortable element less than or equal to $w$. Likewise, there is a related order-preserving map $\pi_{c}^{\uparrow}$ that maps $w$ to the smallest $c$-sortable greater than or equal to $w$. N. Reading showed that the fibers of $\pi_{\downarrow}^{c}$ and $\pi_{c}^{\uparrow}$ are equal and that the fiber containing $w$ is the interval $\left[\pi_{\downarrow}^{c}(w), \pi_{c}^{\uparrow}(w)\right]_{\text {Weak }(W)}$. This turns out to be enough to conclude that the $c$-sortable elements form a lattice quotient of the weak order. Each of these congruences defines an associahedron corresponding to $c$; the 1 -skeletons of these $c$-associahedra are the Hasse diagrams of the $c$-Cambrian lattices.

In contrast, the $m$-eralized $c$-sortable elements no longer form a lattice quotient of Weak ${ }^{(m)}(W)$.

Definition 6.1 The $m$-eralized $c$-Cambrian poset $\operatorname{Camb}_{\text {Sort }}^{(m)}(W, c)$ is the restriction of $\mathrm{Weak}^{(m)}(W)$ to Sort $^{(m)}(W, c)$.

Although we no longer have a lattice quotient, the restriction of Weak ${ }^{(m)}(W)$ to $\operatorname{Sort}^{(m)}(W, c)$ still yields a lattice. This is a generalization of Lemma 3.3 2. Figure 4 shows all 12 st-sorting elements in $\mathrm{Camb}_{\text {Sort }}^{(2)}\left(A_{2}, s t\right)$.

Theorem 6.2 Camb $_{\text {Sort }}^{(m)}(W, c)$ is a sublattice of Weak $^{(m)}(W)$.

\section{$7 m$-eralized Coxeter-sortability and shard intersection order}

Guided by a construction of P. Edelman for classical noncrossing partitions, D. Armstrong defined NC ${ }^{(m)}(W, c)$ as the $m$-multichains in the lattice of noncrossing partitions. On the other hand, N. Reading defined an 


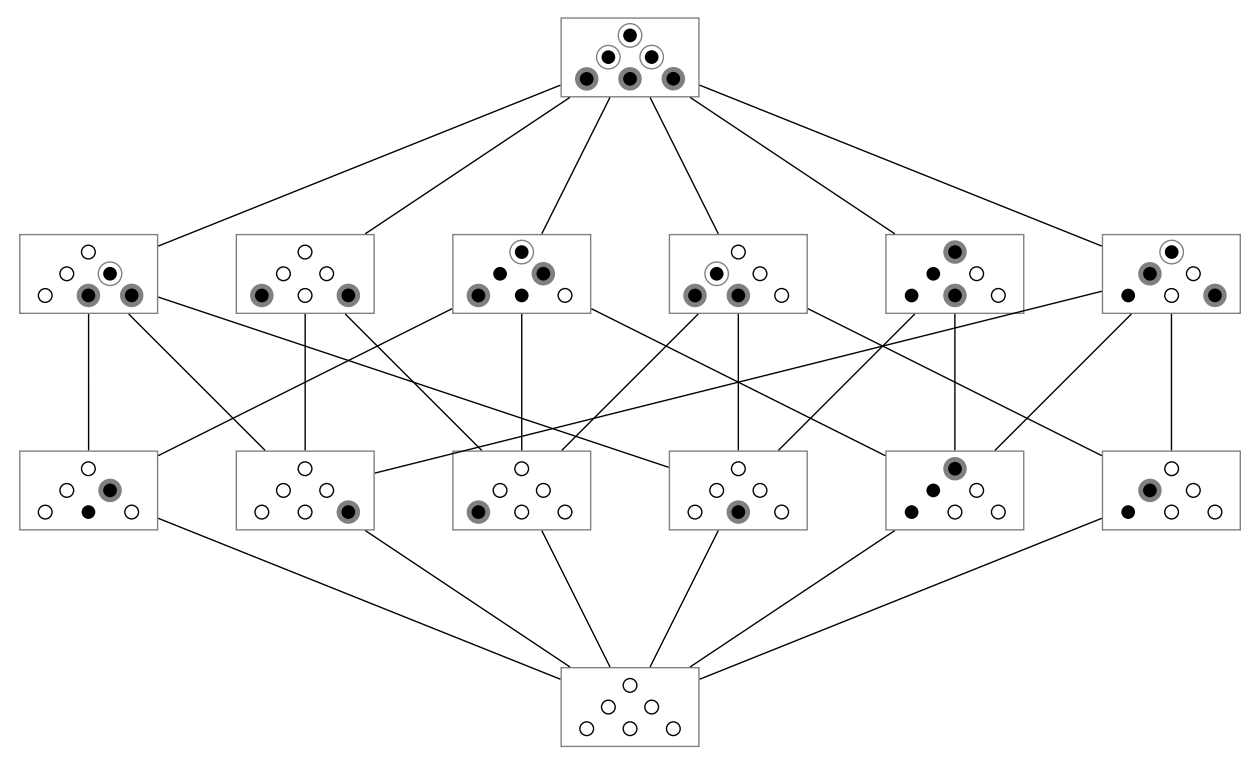

Fig. 5: Shard $\left(A_{3}\right)$ restricted to Sort $\left(A_{3}, s_{1} s_{2} s_{3}\right)$. Sortable elements are given by their inversion sets in the positive roots. Covered reflections are circled in grey, and further inversions in the parabolic subgroup generated by the covered reflections are circled in white.

$\operatorname{order} \operatorname{Shard}(W)$ on the elements of $W$, with the property that the restriction of $\operatorname{Shard}(W)$ to $\operatorname{Sort}(W, c)$ is isomorphic to the noncrossing partition lattice [17]. It is natural to combine D. Armstrong's definition with this isomorphism to $m$-eralize Coxeter-sortable elements as chains of $c$-sortable elements in $\operatorname{Shard}(W)$. In this last section, we provide an alternative characterization of the $m$-sortable elements which has not been considered in the literature before, reconciling our $m$-eralization of $c$-sortable elements with D. Armstrong's $m$-eralization of noncrossing partitions.

In [17], N. Reading defined a delicate slicing procedure on simplicial hyperplane arrangements that cuts hyperplanes into several pieces called shards. The shard intersection order Shard $(W)$ is the set of all intersections of these hyperplane pieces, ordered by reverse inclusion. N. Reading proved that the intersection of the lower shards of an element $w \in W$ is a bijection between $W$ and the set of shard intersections. The longest element $w_{\circ}$ is mapped to the maximal element in the shard intersection order under this bijection. It is possible to define the shard intersection order directly on $W$.

Definition 7.1 Let $u, v \in W$. The shard intersection order $\operatorname{Shard}(W)$ is given by $u \preceq v$ if and only if $\left\langle\operatorname{cov}_{\downarrow}(u)\right\rangle \subseteq\left\langle\operatorname{cov}_{\downarrow}(v)\right\rangle$ and $\operatorname{inv}(u) \subseteq \operatorname{inv}(v)$.

Figure 5 shows Shard $\left(A_{3}\right)$ restricted to $\operatorname{Sort}\left(A_{3}, s_{1} s_{2} s_{3}\right)$.

Proposition 7.2 Definition 7.1 agrees with the definition given in [17. Section 4].

$\mathrm{N}$. Reading showed that the shard intersection order provides an alternative way of thinking about the noncrossing partition lattice $\operatorname{NCL}(W, c)$ : it is the restriction of Shard $(W)$ to the sortable elements $\operatorname{Sort}(W, c)$. 
Theorem 7.3 ([17, Theorem 8.5]) The restriction of $\operatorname{Shard}(W)$ to Sort $(W, c)$ is isomorphic to $\mathrm{NCL}(W, c)$. The isomorphism is given by sending $w \in \operatorname{Sort}(W, c)$ to the product of its covered reflections in the order they appear in $\operatorname{inv}_{\mathcal{R}}(\mathrm{c}(\mathrm{w}))$.

Combining these observations with-D. Armstrong's definition of $m$-noncrossing partitions as $m$-multichains in the noncrossing partition lattice allows us to immediately extract a definition of $m$-sortable elements as $m$-multichains of sortable elements in $\operatorname{Shard}(W)$.

Definition 7.4 Let Sort $_{\text {shard }}^{(m)}(W, c)$ be the set of m-multichains of c-sortable elements in Shard $(W)$,

$$
\operatorname{Sort}_{\text {shard }}^{(m)}(W, c):=\left\{\left(w_{1} \succeq w_{2} \succeq \cdots \succeq w_{m}\right): w_{i} \in \operatorname{Sort}(W, c)\right\} .
$$

Theorem 7.5 There is an explicit bijection $\operatorname{Sort}_{\text {shard }}^{(m)}(W, c) \stackrel{\sim}{\longrightarrow} \operatorname{Sort}^{(m)}(W, c)$.

Remark 7.6 In analogy to componentwise absolute order on $\mathrm{NC}_{\delta}^{(m)}(W, c)$-as considered by D. Armstrong in [2]-by Theorems 5.4 and 7.5. componentwise weak order on $\operatorname{Sort}_{\text {shard }}^{(m)}(W, c)$ defines the $m$ eralized c-Cambrian lattice.

Example 7.7 For type $A_{3}$ with $m=2$ and $c=s_{1} s_{2} s_{3}$, consider the chain of sortable elements in shard order

$$
\left(w_{1} \succeq w_{2}\right)=\left(s_{1} s_{2} s_{3} s_{2} \succeq s_{1} s_{2} s_{3}\right) \in \operatorname{Sort}_{\text {shard }}^{(m)}\left(A_{3}, c\right) .
$$

We now compute the bijection of Theorem 7.5.

$$
\operatorname{inv}_{\mathcal{R}}\left(w_{1}\right)=\{(12),(13),(14),(34)\} \supseteq\{(12),(13),(14)\}=\operatorname{inv}_{\mathcal{R}}\left(w_{2}\right) .
$$

Note that $w_{1}$ has covered reflections $\{(13),(34)\}$ and associated simple reflections $\left\{s_{3}, s_{2}\right\}$, since $s_{3}^{w_{1}}=$ $(34)^{w_{1}}=(13)$ and $s_{2}^{w_{1}}=(23)^{w_{1}}=(34)$. The covered reflections generate the nonstandard parabolic subgroup $W_{\text {cov }_{\downarrow}\left(w_{1}\right)}$ containing the reflections $\{(13),(14),(34)\}$. Restricting to $W_{\text {cov }_{\downarrow}\left(w_{1}\right)}, w_{2}$ is sent to the element in $W_{\operatorname{cov}_{\downarrow}\left(w_{1}\right)}$ with reflection inversion set $\{(13),(14)\}$. (Note that we can uniquely recover $\operatorname{inv}_{\mathcal{R}}\left(w_{2}\right)$ by adding in missing initial segments of oriented dihedral subgroups - in this case, the dihedral generated by $\left\{s_{1}, s_{2}\right\}$ forces $(1,2)$ to be added back to the inversion set). We conjugate these inversions by $w_{1}^{-1}$ to pass to the corresponding standard parabolic subgroup $W_{\operatorname{des}_{R}\left(w_{1}\right)}$, obtaining the inversion set $\{(34),(24)\}$. This inversion set corresponds to an element with reduced word $s_{3} s_{2}$. Therefore, $\left(s_{1} s_{2} s_{3} s_{2} \succeq s_{1} s_{2} s_{3}\right)$ is mapped to the Garside factorization $s_{1} s_{2} s_{3} s_{2} \cdot s_{3} s_{2}$.

\section{Acknowledgements}

We thank Drew Armstrong, Nathan Reading, and Vic Reiner for many helpful discussions.

\section{References}

[1] D. Armstrong, B. Rhoades, and N. Williams. Rational associahedra and noncrossing partitions. Electron. J. Combin., 20(3), 2013.

[2] Drew Armstrong. Generalized noncrossing partitions and combinatorics of Coxeter groups. Memoirs of the American Math. Society, 202(949), 2006. 
[3] David Bessis. The dual braid monoid. Annales scientifiques de l'Ecole normale supérieure, 36(5):647-683, 2003.

[4] Anders Björner and Michelle Wachs. Shellable nonpure complexes and posets. II. Transactions of the American Math. Society, 349(10):3945-3975, 1997.

[5] Egbert Brieskorn and Kyoji Saito. Artin-gruppen und Coxeter-gruppen. Inventiones mathematicae, 17(4):245-271, 1972.

[6] Aslak Buan, Idun Reiten, and Hugh Thomas. From $m$-clusters to $m$-noncrossing partitions via exceptional sequences. Mathematische Zeitschrift, 271(3-4):1117-1139, 2012.

[7] Cesar Ceballos, Jean-Philippe Labbé, and Christian Stump. Subword complexes, cluster complexes, and generalized multi-associahedra. J. Algebraic Combin., 39(1):17-51, 2014.

[8] Patrick Dehornoy. Combinatorics of normal sequences of braids. J. of Combin. Theory, Series A, 114(3):389-409, 2007.

[9] Paul Edelman. Chain enumeration and non-crossing partitions. Discrete Math., 31(2):171-180, 1980.

[10] Sergey Fomin and Nathan Reading. Generalized cluster complexes and Coxeter combinatorics. International Math. Research Notices, 44:2709-2757, 2005.

[11] Sergey Fomin and Andei Zelevinsky. Cluster algebras II: finite type classification. Invent. Math., 154:63-121, 2003.

[12] Colin Ingalls and Hugh Thomas. Noncrossing partitions and representations of quivers. Compos. Math, 145:1533-1562, 2009.

[13] Vincent Pilaud and Christian Stump. Brick polytopes of spherical subword complexes and generalized associahedra. 276, 2015. Adv. Math.

[14] Nathan Reading. Cambrian lattices. Advances in Math., 205(2):313-353, 2006.

[15] Nathan Reading. Clusters, Coxeter-sortable elements and noncrossing partitions. Transactions of the American Math. Society, 359:5931-5958, 2007.

[16] Nathan Reading. Sortable elements and Cambrian lattices. Algebra Universalis, 56(3-4):411-437, 2007.

[17] Nathan Reading. Noncrossing partitions and the shard intersection order. Journal of Algebraic Combin., 33(4):483-530, 2011.

[18] Nathan Reading and David Speyer. Sortable elements in infinite Coxeter groups. Transactions of the American Math. Society, 363(2):699-761, 2011.

[19] Victor Reiner. Non-crossing partitions for classical reflection groups. Discrete Math., 177(1):195222, 1997.

[20] Christian Stump, Hugh Thomas, and Nathan Williams. Cataland: Why the Fuss? arXiv preprint arXiv:1503.00710, 2015. 